\title{
COMPARATIVE STUDY OF CLINICAL VERSUS USG IN DIAGNOSIS OF EARLY TRIMESTER BLEEDING PV
}

\section{Gynecology}

Dr.Narendra Nath Assistant Professor, Department of Gynaecology and Obstetrics, Midnapore Medical Hait

Dr.Brahmarshi Associate Professor, Department of Biochemistry, Midnapore Medical College and
Das* Hospital, Paschim Medinipur,Pin - 721101.*Corresponding Author

Dr.Ratan Chandra Associate Professor, Department of Gynaecology and Obstetrics, Midnapore Medical Mandal College and Hospital, Paschim Medinipur,Pin - 721101.

\begin{tabular}{ll}
\hline $\begin{array}{l}\text { Dr. Haricharan } \\
\text { Roy }\end{array}$ & $\begin{array}{l}\text { Assistant Professor. Department of Gynaecology and Obstetrics, Midnapore Medical } \\
\text { College and Hospital, Paschim Medinipur,Pin - 721101. }\end{array}$ \\
\hline Dr. Debarshi Jana & IPGMER and SSKM Hospital, Kolkata \\
\hline
\end{tabular}

\section{ABSTRACT}

Background: Threatened abortion is till most common cause of early trimester bleeding PV and can be diagnosed and managed by early USG diagnosis. Materials and methods: This was a prospective observational study. Place of the study was Department of Obstetrics and Gynaecology and Department of Radiodiagnosis, Midnapore Medical College and Hospital from January 2019 to June 2020. Eighteen months. Result: When the clinical method to diagnose threatened miscarriage was compared to the sonographic method, it was evident that sonographic method was reliable than the clinical method and the difference was statistically significant. Conclusion: In case of missed miscarriage and complete miscarriage, although the percentage of discrepancy was $100 \%$, on further statistical analysis, the discrepancy was not statistically significant. And the cause behind this was probably inadequate sampling.

\section{KEYWORDS}

\section{EARLY TRIMESTER BLEEDING PV, Clinically, USG}

\section{INTRODUCTION}

Aronu et al ${ }^{1}$ performed a retrospective study to evaluate the accuracy of clinical diagnosis of first trimester vaginal bleeding in comparison with ultrasonography. This study was performed because of the high incidence of bleeding per vaginum in first trimester pregnancy and accurate diagnosis was very necessary to enable the clinician to institute appropriate and prompt treatment. A total of 101 women participated in the study. The mean age was 30.2 years. The most common clinical diagnosis was threatened abortion $(65.3 \%)$ which was reduced to $34.7 \%$ by ultrasonography. The overall concordance was $44.55 \%$. the highest concordance of $66.7 \%$ was observed in the ectopic pregnancy group. The authors concluded that ultrasound should be done early in patients with first trimester bleeding as this would lead to early definitive diagnosis and commencement of appropriate therapeutic intervention aimed at reduction of mortality and morbidity, reduced hospital stay and cost.

Kirk $^{2}$ opined that the majority of ectopic pregnancies can be diagnosed on transvaginal scan before treatment. Diagnosis should be based on positive visualization of an ectopic pregnancy rather the inability to visualize an intrauterine pregnancy. The majority of ectopic pregnancies will be visible on the initial transvaginal scan performed. Those who do not have their ectopic pregnancies visualized on the first examination will be initially classified as having a pregnancy of unknown location. They will then need follow-up until a diagnosis of ectopic pregnancy is confirmed.

Al Meymar et al ${ }^{3}$ published a review paper on the role of ultrasonography in the diagnosis and management of early pregnancy complications and concluded that transvaginal ultrasonography was the cornerstone of the management of women with early pregnancy complications. Furthermore, a clear understanding of the behaviour of serum hCG and progesterone is a requirement for those working in the field of early pregnancy care. The authors also stressed the fact to remember that we treat women and not ultrasound scans, therefore the clinical status of the women was of paramount importance.

Paspulati et al ${ }^{4}$ in their study found that vaginal bleeding was a leading cause of presentation for emergency care during the first trimester of the pregnancy. Clinical assessment of the pregnancy outcome at this stage was less reliable. Ultrasonographic examination, in their opinion, was crucial in establishing intrauterine pregnancy (IUP) and early pregnancy failure and to exclude other causes of bleeding, such as ectopic pregnancy and molar pregnancy. Diagnosis of a normal IUP at this stage not only assists the physician in an expectant management, but also gives a psychologic boost to the patient. Yadav et al performed a descriptive study involving 100 low-risk pregnant women between 8 to 12 weeks of gestation with known last menstrual period conducted at Subharti Medical College, Meerut. All the patients were subjected to transabdominalsonography (TAS) and followed by transvaginalsonography (TVS) and were followed till delivery with subsequent scan when necessary. A total of 100 pregnancies were intrauterine of which 3 were anembryonic and 4 had early pregnancy failure, 13 pregnancies were re-dated, one with subchorionic leave separation, 3 physiological herniation of bowel loops, and 1 physiological herniation with single cord cyst were identified only by TVS and had favourable outcome. Two twin pregnancies were identified and managed. The study showed the routine ultrasound in first trimester in low-risk women was helpful in timely identification of complications and re-dating of the pregnancies, thereby reducing the induction rates. This study also gives insight that TVS is superior to TAS in evaluation of foetal structure.

Kurmi et al ${ }^{6}$ performed that a total of 100 women were enrolled in the study. The authors concluded that, bleeding per vaginum in the first trimester was one of the most common causes for emergency admission to the obstetric department. Ultrasonographic examination was a non-invasive, no-ionizing and easily available method to assess patients with first trimester bleeding. Ultrasonography was highly accurate in diagnosing actual causes of bleeding and guiding the clinician to choose the appropriate line of management.

Dr. M Ayub Hassan ${ }^{7}$ concluded in his review article that, ultrasound was the best modality to evaluate the first trimester pregnancy complications especially when was done trans-vaginally.

Condous et al ${ }^{8}$ in their study concluded that the majority of women with a miscarriage can be offered expectant management with a reasonable prospect of success and with no increase in the complication rate. Approximately $90 \%$ of incomplete miscarriages and $50 \%$ of missed miscarriages and anembryonic pregnancies could be expected to miscarry within 2 weeks. The odds of a woman completing her miscarriage with each subsequent week diminished with time. Most women would opt for surgical intervention if they had 
not completed their miscarriage within the 2-week window. The data suggested that the ultrasound appearances of the endometrial cavity contents bore no relation to the outcome of expectant management. The antero-posterior diameter of the endometrial cavity did not relate to the odds of successful completion. The authors concluded that, there was little sense in repeating ultrasound scans as part of follow-up protocols as the endometrial cavity does not relate to the odds of successful completion. On this basis there seemed little sense in repeating ultrasound scans as part of follow-up protocols as the endometrial cavity contents do not reflect the likelihood of success. Resources for follow-up might be better orientated towards counselling for early pregnancy loss rather than medical intervention. The need for surgery can be based on the presence or absence of pain and bleeding as well as clinical evidence of infection. The success of expectant management for missed and anembryonic pregnancies was significantly less than for incomplete miscarriage. Medical management was more likely to play a role in those women.

Murugan et al ${ }^{9}$ concluded in their review article that, trans-vaginal ultrasound was the modality of choice for establishing the presence of an intrauterine pregnancy, evaluating pregnancy viability, gestational age and multiplicity; detecting pregnancy related complications and diagnosing ectopic pregnancy.

\section{MATERIALSAND METHODS}

PLACE OF STUDY: Department of Obstetrics and Gynaecology and Department of Radiodiagnosis, Midnapore Medical College and Hospital from January 2019 to June 2020. Eighteen months.

STUDY POPULATION: All pregnant women admitted in our hospital with early pregnancy complications (less than 13 weeks' gestation).

SAMPLE SIZE: The sample size was 100

INCLUSION CRITERIA: Any pregnant woman with less than 13 weeks' gestation admitted with vaginal bleeding and/or pain abdomen.

\section{EXCLUSION CRITERIA}

- Pregnancy beyond 13 weeks.

- Non-obstetric vaginal bleeding.

- Bleeding following medical/surgical termination of pregnancy.

\section{RESULT AND ANALYSIS}

The women recruited in the study were divided in 3 groups according to age. Group 1 ( 16 to 20 years) consisted of $73(73 \%)$ mothers, group (21-25 years) consisted of 23 (23\%) mothers and group 3 (26 to 30 years) consisted of $4(4 \%)$ mothers. As expected, majority of the study population belonged to rural area $(63 \%)$. Only $37(37 \%)$ women belonged to urban area. Majority of the mothers enrolled in the study were literate. Sixty-five women of the total 100 women enrolled in the study were primary school pass outs. Rest were secondary school (16) and college (9) educated. Ninety-seven $(97.0 \%)$ patients were housewives and 3(3.0\%) patients were teachers.

We found that eighty-nine $(89 \%)$ patients were from low socioeconomic status and $11(11 \%)$ patients belonged to middle socioeconomic status. Among the 100 women enrolled in the study, 74 (74\%) women were of normal BMI. Twenty-one $(21 \%)$ women were underweight, $2(2 \%)$ were overweight and $3(3 \%)$ were obese. Majority of the mothers $(61 \%)$ enrolled in the study were un-booked to ante-natal care at the time of presentation to the emergency department. Forty-four (44\%) patients had P0+0 parity, $14(14 \%)$ patients had $\mathrm{P} 0+1$ parity, $3(3 \%)$ patients had $\mathrm{P} 0+2$ parity, $6(6 \%)$ patients had P0+1parity, $6(6 \%)$ patients had $\mathrm{P} 1+0$ parity, $12(12 \%)$ patients had $\mathrm{P} 1+1$ parity, $9(9 \%)$ patients had $\mathrm{P} 2+0$ parity and $6(6 \%)$ patients had $\mathrm{P} 3+1$ parity.

Our study showed that three (3\%) patients had 5 weeks of gestational age, $5(5 \%)$ patients had 6 weeks of gestational age, $24(24 \%)$ patients had 7 weeks of gestational age, $36(36 \%)$ patients had 8 weeks of gestational age, $15(15 \%)$ patients had 9 weeks of gestational age, $1(1 \%)$ patient had 10 weeks of gestational age, $8(8 \%)$ patients had 11 weeks of gestational age, $5(5 \%)$ patients had 12 weeks of gestational age and $3(3 \%)$ patients had 13 weeks of gestational age. Sixty-six $(66 \%)$ patients presented with bleeding per vaginum and $34(34 \%)$ patients presented with abdominal pain. Eighty-two $(82 \%)$ patients had threatened abortion as the clinical diagnosis. Only one mother was clinically diagnosed to be a case of missed abortion. Clinically $9(9 \%)$ mothers were diagnosed as cases of incomplete miscarriage. Complete miscarriage was the clinical diagnosis in $4(4 \%)$ mothers.

We found that ectopic pregnancy was clinically diagnosed in 3 mothers (3\%) among the study population. On sonographic examination, 45 $(45 \%)$ women were diagnosed as cases of threatened miscarriage. Sonographically, missed miscarriage was diagnosed in $22(22 \%)$ women. Incomplete miscarriage was the sonographic diagnosis in 10 $(10 \%)$ women. Complete miscarriage was the sonographic diagnosis in $11(11 \%)$ women Sonographically, $7(7 \%)$ mothers were diagnosed as cases of molar pregnancy. Sonographically, $5(5 \%)$ mothers were diagnosed as cases of ectopic pregnancy. When the 82 mothers diagnosed clinically to be suffering from threatened abortion were examined sonographically, only $45(54.88 \%)$ women were confirmed to be cases of threatened miscarriage. Rest of them were cases of missed miscarriage [17 (20.73\%)], incomplete miscarriage [4 $(4.88 \%)]$, complete miscarriage [9 $(10.98 \%)]$, molar pregnancy [5 $(6.09 \%)]$ and ectopic pregnancy [2 (2.44\%)].

The only mother who was diagnosed to be having missed miscarriage clinically was sonographically confirmed to be a case of complete miscarriage. Among the 9 cases of incomplete miscarriage as clinical diagnosis, only $5(11.11 \%)$ were confirmed to be cases of incomplete miscarriage sonographically. Rest were cases of, missed miscarriage [2 (22.22\%], complete miscarriage [1 (11.11\%] and molar pregnancy [1 (11.11)]. Complete miscarriage was the clinical diagnosis in 4 women. On sonographic examination, none of them were cases of complete miscarriage. Three mothers $(75 \%)$ were diagnosed to be cases of missed miscarriage and $1(25 \%)$ was diagnosed to be suffering from incomplete miscarriage. Molar pregnancy was the clinical diagnosis in only one mother and on sonographic examination she was confirmed to be a case of molar pregnancy. The three cases of ectopic pregnancy diagnosed clinically were confirmed sonographically to be cases of ectopic pregnancy. Among the 100 women recruited in the study, clinically 82 women were diagnosed to be cases of threatened miscarriage. But, when subjected to sonographic examination, only 45 women were confirmed to be cases of threatened miscarriage. So, the accuracy of clinical method was $54.88 \%$ and discrepancy was $45.12 \%$. Our study showed that in one case diagnosed clinically as missed miscarriage was refuted by sonographic examination and was diagnosed as complete miscarriage. Out of the 9 cases diagnosed clinically to be cases of incomplete miscarriage, 5 cases were confirmed by sonography. So, the accuracy of clinical examination to diagnose incomplete miscarriage was $55.55 \%$. The accuracy of clinical method to diagnose ectopic pregnancy and molar pregnancy was $100 \%$ in our study. When the clinical method to diagnose threatened miscarriage was compared to the sonographic method, it was evident that sonographic method was reliable than the clinical method and the difference was statistically significant. When the clinical method to diagnose missed miscarriage was compared with the sonographic method, the difference was not statistically significant. This might be due to inadequate sample size. As far as the diagnosis of incomplete miscarriage was concerned, sonographic method was expectedly superior to clinical method.

While diagnosing complete miscarriage, clinical method was comparable to sonographic method. The association of complete miscarriage clinical diagnosis versus complete miscarriage USG diagnosis was not statistically significant $(\mathrm{p}=0.3610)$. This might be due to inadequate sampling. While diagnosing molar pregnancy, ultrasonography was expectedly superior to clinical method. The association of molar pregnancy clinical diagnosis vs molar pregnancy USG diagnosis was statistically significant $(p=0.0002)$. The association of ectopic pregnancy clinical diagnosis vs ectopic pregnancy USG diagnosis was statistically significant $(p<0.0001)$. Management of the women enrolled to the study was based on the sonographic diagnosis. The women with sonographic diagnosis of threatened abortion were followed up by routine antenatal care. Cases of incomplete miscarriage were managed by dilatation and evacuation. Cases of molar pregnancy were management suction-evacuation. Ectopic pregnancy was managed by laparotomy followed by salpingectomy.

\section{DISCUSSIONAND CONCLUSION}

BACKGROUND DEMOGRAPHIC FEATURES

The mothers with early pregnancy complications enrolled in the study were evaluated regarding their demographic features. 
Majority of the women $(60 \%)$ enrolled in the study were below 20 years of age. Most of them, i.e. $63 \%$ of the mothers, were from rural areas. Sixty five percent of the mothers received primary level of school education. Ninety seven percent of the mothers were housewives and $89 \%$ of the mothers belonged to the low socioeconomic status. As far as ANC status was concerned, $61 \%$ of the mothers were not yet booked for ANC at the time of their enrolment in the study and it was quite expected as in rural areas it is not very common to get booked for ANC in the first trimester.

\section{PRESENTING COMPLAINTS:}

The most common complaint the mothers were admitted with was bleeding per vaginum. Sixty-five mothers $(65 \%)$ presented with bleeding per vaginum and the rest with pain abdomen $(35 \%)$.

CLINICALDIAGNOSES AND SONOGRAPHIC DIAGNOSES:

The most common clinical diagnosis was threatened abortion among the women enrolled in the study. A total of $82(82 \%)$ women were diagnosed clinically to be cases of threatened miscarriage. When those women were subjected to sonographic examination to confirm the clinical diagnosis, only 45 women $(54.88 \%)$ were diagnosed with threatened miscarriage. Rest were diagnosed as follows- missed miscarriage $17(20.73 \%)$, incomplete miscarriage $4(4.88 \%)$, complete miscarriage $9(10.98 \%)$, molar pregnancy $5(6.09 \%)$ and ectopic pregnancy 2 (2.44\%).

The second most common clinical diagnosis was incomplete miscarriage. A total of 9 women were diagnosed with incomplete miscarriage clinically. However, when sonographic confirmation was done, $5(55.55 \%)$ women were diagnosed to be suffering from incomplete miscarriage and the rest were diagnosed with missed miscarriage [2 (22.22\%)], complete miscarriage [1 (11.11\%)], molar pregnancy $[1(11.11 \%)]$.

Clinically, complete miscarriage was diagnosed in 4 mothers (4\%). And after sonographic confirmation, the diagnoses were as follows, missed miscarriage [3 (75\%)] and incomplete miscarriage [1 (25\%)].

Clinically 3 women were diagnosed with ectopic pregnancy and all three were confirmed to be cases of ectopic pregnancy on ultrasonography.

Molar pregnancy was clinically diagnosed in 1 woman and the diagnosis was confirmed by ultrasonography.

Missed miscarriage was diagnosed in 1 woman and when she was subjected to ultrasonographic examination she was diagnosed to be a case of complete miscarriage.

When the overall clinical and sonographic diagnoses were put to further statistical analysis, the following information were obtained. In our study we found out that, among the women enrolled in the study, ultrasonography was superior to clinical methods to diagnose threatened miscarriage, incomplete miscarriage, molar pregnancy, ectopic pregnancy.

\section{REFERENCE}

1. Aronu ME et al. A Review of the Correlation between Clinical Diagnosis in First Trimester Vaginal Bleeding. Ann Med Health Sci Res 2018; 8: 120-4.

2. Kirk E. Ultrasound in the diagnosis of ectopic pregnancy. Clin Obstet Gynecol 2012; 55:395-401.

3. 37. Al-Memar M, Kirk E, Bourne T. The role of ultrasonography in the diagnosis and management of early pregnancy complications. The Obstetrician \& Gynaecologist $2015 ; 17: 173-81$.

4. 38. Paspualti RM, Bhatt S, Nour SG. Sonographic estimation of first trimester bleeding. Radiol Clin North Am 2008 Mar;46(2):437.

5. 39. Yadav S, Neeraj. Role of routine ultrasound in first trimester of pregnancy: A 39. Yadav S, Neeraj. Role of routine ultrasound
descriptive study. Int J Sci Stud 2017;4(10):125-8.

6. 40. Kurmi D, Jadhav V, Misri A. Role of pelvic sonography in first trimester bleeding. Journal of Evolution of Medical and Dental Sciences 2015; 4(49): 8516-25.

7. 41. M Ayub Hussain. "First Trimester Pregnancy Complications how could be Evaluated". EC Gynaecology 7.6 (2018): 241-56.

8. 42. Condous G, Okaro E, Bourne T. The conservative management of early pregnancy complications: A review of the literature. Ultrasound Obstet Gynecol 2003;22: 420-30.

9. 43. Murugan VA, Murphy RO, Dupuis C, Goldstein A, Kim YH. Role of ultrasound in the evaluation of first trimester pregnancies in acute setting. Ultrasonography $2020 \mathrm{Apr}$; $39(2): 178-89$ 